\title{
Antimicrobial activity of pure platelet-rich plasma against microorganisms isolated from oral cavity
}

\author{
Lorenzo Drago ${ }^{1,2^{*}}$, Monica Bortolin ${ }^{3}$, Christian Vassena ${ }^{1}$, Silvio Taschieri ${ }^{3}$ and Massimo Del Fabbro ${ }^{3}$
}

\begin{abstract}
Background: Autologous platelet concentrates (PCs) have been extensively used in a variety of medical fields to promote soft and hard tissue regeneration. The significance behind their use lies in the abundance of growth factors in platelets a-granules that promotes wound healing. In addition, antibacterial properties of PCs against various bacteria have been recently pointed out. In this study, the antimicrobial effect of pure platelet-rich plasma (P-PRP) was evaluated against oral cavity microorganisms such as Enterococcus faecalis, Candida albicans, Streptococcus agalactiae, Streptococcus oralis and Pseudomonas aeruginosa. Blood samples were obtained from 17 patients who underwent oral surgery procedures involving the use of P-PRP. The antibacterial activity of P-PRP, evaluated as the minimum inhibitory concentration (MIC), was determined through the microdilution twofold serial method.
\end{abstract}

Results: P-PRP inhibited the growth of Enterococcus faecalis, Candida albicans, Streptococcus agalactiae and Streptococcus oralis, but not of Pseudomonas aeruginosa strains.

Conclusions: P-PRP is a potentially useful substance in the fight against postoperative infections. This might represent a valuable property in adjunct to the enhancement of tissue regeneration.

Keywords: Platelet concentrate, Oral infection, Antimicrobial effect, Minimum inhibitory concentration, Oral cavity

\section{Background}

In the past 20 years, the use of autologous platelet concentrates (PCs) has gained great popularity in a variety of medical fields such as dentistry, oral surgery, orthopedics, sports medicine, dermatology, ophthalmology, cosmetic and plastic surgery. The rationale for their use stems from the fact that platelets store and release, upon activation, growth factors such as PDGF, TGF- $\beta$, EGF, VEGF, IGF-1, FGF, HGF and other molecules that modulate the wound healing response in both hard and soft tissues. In addition, anti-inflammatory properties of PCs have been pointed out associated with a marked reduction of postoperative pain and swelling [1-3].

Recently, the clinical and in vitro antibacterial effect of human PCs has been reported against bacteria such as Staphylococcus aureus, Staphylococcus epidermidis,

\footnotetext{
* Correspondence: lorenzo.drago@unimi.it

${ }^{1}$ Laboratory of Clinical Chemistry and Microbiology, I.R.C.C.S. Galeazzi

Orthopaedic Institute, Via R. Galeazzi 4, Milan 20161, Italy

${ }^{2}$ Department of Biomedical Sciences for Health, University of Milan, Milan, Italy

Full list of author information is available at the end of the article
}

Escherichia coli and Klebsiella pneumoniae while no activity has been found against Enterococcus faecalis, Pseudomonas aeruginosa, Enterobacter cloacae, Bacillus cereus and Bacillus subtilis [4-12].

The mechanism of the antibacterial effect of PCs is not yet fully understood. Existing evidence suggests that platelets may play multiple roles in antimicrobial host defense: they generate oxygen metabolites, including superoxide, hydrogen peroxide and hydroxyl free radicals; [13-15] they are capable of binding, aggregating, and internalizing microorganisms, which enhances the clearance of pathogens from the bloodstream; they participate in antibody-dependent cell cytotoxicity functions to kill protozoal pathogens; finally, platelets release an array of potent antimicrobial peptides [16,17].

Several techniques are available for the production of PCs, leading to products with different biological characteristics. The various PCs can be classified into four main categories, depending on their leucocyte and fibrin content: pure platelet-rich plasma (P-PRP), pure platelet-rich fibrin (P-PRF), leukocyte- and platelet-rich plasma (L-PRP) and leukocyte- and platelet-rich fibrin 
(L-PRF). [18] L-PRP and L-PRF might contain substantial amount of white blood cells. The respective effects of platelets and leucocytes in PCs have not been elucidated yet, and the contribution of leucocytes to the observed overall effect remains unclear [19]. Therefore in this study we decided to use a widely documented technology developed in 1999 by Anitua that allows the production of leukocyte-poor platelet concentrate [20].

The aim of this study was to evaluate in vitro the antibacterial effect of P-PRP against microorganisms colonizing the oral cavity such as Enterococcus faecalis, Candida albicans, Streptococcus agalactiae, Streptococcus oralis and Pseudomonas aeruginosa.

\section{Methods \\ Donors}

Blood samples were obtained from 17 adult patients (two men, 15 women; mean age $59 \pm 11$ years, age range 34-75 years) who underwent oral surgery procedures (dental implant placement, tooth extraction) involving the use of P-PRP. All subjects were in general good health (ASA 1-2). No patient took antibiotics during the month before surgery, nor was under anticoagulant or immunosuppressive therapy. Written informed consent for participation in the study was obtained from all patients. The present research was performed within the guidelines of the Helsinki Declaration for biomedical research involving human subjects. The study was approved by the Review Board of the Galeazzi Orthopedic Institute.

\section{Blood collection and production of P-PRP}

Fresh human whole blood from donors was processed using $\mathrm{PRGF}^{\circledR}$ System IV (BTI, Biotechnology Institute, Vitoria, Alava, Spain) to create a platelet concentrate, according to manufacturer's protocol. Briefly, peripheral blood from each donor was taken by venipuncture into $5 \mathrm{ml}$ blood-collecting tubes with 3,8\% (wt/vol) trisodium citrate as anticoagulant. Blood was centrifuged at $460 \mathrm{~g}$ for $8 \mathrm{~min}$ at room temperature. After centrifugation, 3 components were obtained: red blood cells, a thin layer of leukocytes referred to as "buffy coat" and plasma. The $1 \mathrm{ml}$ plasma fraction located above the red cell fraction, but not including the buffy coat, was collected.

\section{Determination of platelet and leukocyte count}

Platelet concentration in whole blood and P-PRP was counted automatically using a hematology analyzer (Sismex XE-2100, Norderstedt, GER). To evaluate the purity of P-PRP, we have also performed a white blood cells count both in whole blood and P-PRP. According to Anitua et al. [8], leukocyte levels in P-PRP must be lower than in whole blood $\left(<10^{3} / \mu \mathrm{l}\right)$.

\section{Activation of P-PRP}

P-PRP was activated shortly before use. In order to initiate clotting and trigger the release of platelet content, $\mathrm{CaCl} 2$ was added ( $50 \mu \mathrm{l}$ per $\mathrm{ml}$ of P-PRP).

\section{Bacterial strains}

Clinical isolates collected from patients with oral and dental infectious diseases have been used.

Microorganisms were stored at $-80^{\circ} \mathrm{C}$ before analysis. In particular, we selected the most representative microorganisms colonizing and affected the oral cavity belonging to gram positive, gram negative and fungi, such as E. faecalis (3 vancomycin-sensitive enterococcus (VSE) and 2 vancomycin-resistant enterococcus (VRE)), C. albicans, S. agalactiae, $S$. oralis and $P$. aeruginosa. This strains were previously identified by biochemical identification (API system and Vitek2 Compact, Biomerieux, Marcy l'Etoile, France) and confirmed by DNA sequencing of about $80 \mathrm{pb}$ of variable regions V1 and V3 of the $16 \mathrm{~S}$ rRNA gene by Pyrosequencing (PSQ96RA, Diatech, Jesi, Italy). For each species, we used five strains isolated from different patients that presented dental abscesses. Each strain presented different characteristics (e.g. different antibiotic resistance). In addition, ATCC strains were used as control: E. faecalis ATCC \#29212, C. albicans ATCC \#928, S. agalactiae ATCC \#13813, S. oralis ATCC \#35037 and P. aeruginosa ATCC \#27853.

Before use, strains were thawed and reconstituted in appropriate medium (e.g. Brain Heart Infusion broth (BHI; Biomerieux, Marcy l'Etoile, France) additioned with $5 \%$ defibrinated blood) at $37^{\circ} \mathrm{C}$ for 24 hours.

\section{Determination of antibacterial activity}

The minimum inhibitory concentration (MIC), defined as the lowest concentration of an antimicrobial substance that will inhibit the visible growth of a microorganism, was determined by broth microdilution method.

After seeding in appropriate medium (Trypticase Soy Agar or Columbia Blood Agar; Biomerieux, Marcy l'Etoile, France), a suspension in BHI was prepared for each strain, with an optical density equal to $0,5 \mathrm{McF}$ arland $\left(1 \times 10^{8} \mathrm{CFU} / \mathrm{mL}\right)$. After obtaining a concentration of $1 \times 10^{4} \mathrm{CFU} / \mathrm{mL}$ using appropriate dilutions, $10 \mu \mathrm{l}$ of each suspension were inoculated in a 96-wells microplate containing $100 \mu \mathrm{l}$ of $\mathrm{BHI}$ and a serial dilution of activated P-PRP. In order to asses that the bacteria and yeast have not responding in an unexpected manner, for each strain, we have performed a positive control which consists in inoculate the bacterial suspension in BHI without P-PRP. After incubation at $37^{\circ} \mathrm{C}$ for 24 hours, MIC values were read. MIC values correspond to the concentration of $\mathrm{P}$ PRP present in the last well in which a bacterial growth is observable. The assay was performed in duplicate for each strain and, if the two MIC differed by more than two 
wells, the assay was repeated. Results were expressed as mean \pm standard deviation.

A minimum bactericidal concentration (MBC) test was also performed. $\mathrm{MBC}$ is the lowest concentration of a substance required to kill a particular bacterium. It was determined from broth microdilution MIC tests by subculturing $100 \mu \mathrm{l}$ of bacterial suspension to agar media.

\section{Results}

As expected, the P-PRP produced was leukocyte-depleted $(0,34 \pm 0,27) \times 10^{3} / \mu \mathrm{l}$. In order to obtain the minimum platelet concentration ranges of P-PRP capable of inhibiting bacterial growth, we calculated the mean MIC of the 5 strains tested for each microorganism.

Values are presented in Table 1. MIC are expressed as number of platelets/ $\mu$ l. As can be seen from the data, the platelet concentration ranges are fairly uniform among microorganisms, except for $C$. albicans, whose range of MIC is about twice the others, and for $P$. aeruginosa, which is not inhibited by P-PRP. S. oralis seems to be more sensible than other bacteria to the antibacterial activity of P-PRP. No differences were observed between E. faecalis VRE and E. faecalis VSE regarding susceptibility to P-PRP.

MIC values observed for ATCC bacterial strains fell into the same platelet concentration ranges as those of the corresponding clinical isolates.
MBC tests showed that $C$. albicans was never killed by P-PRP, while the other microorganisms were killed at concentrations 3-4 times the MIC.

\section{Discussion}

The regenerative potential of PCs has been explored considerably during the last two decades. On the contrary, in the available literature only few reports can be found about their antimicrobial effects.

To date, the components responsible for the antimicrobial activity of PCs remain poorly understood, in particular because these materials are a complex mixture of platelets, white blood cells and plasma. The respective impact of the plasma and cellular components has not been studied in detailyet. Several antimicrobial factors have been proposed, including platelet antimicrobial proteins and peptides of the innate immune defense, or platelet $\alpha$-granules components, such as complement and complement-binding proteins. [17,21-26] Direct interaction of platelets with microorganisms and participation in antibody-dependent cell cytotocity and white blood cells in direct bacterial killing, release of myeloperoxidas, activation of the antioxidant responsive element and antigen-specific immune response have also been suggested. [12,15,27] The role of leucocytes within PCs is a matter of intense debate. Some authors have suggested that inclusion of white blood cells in PCs may help to improve the stability of the scaffold and increase the antimicrobial

Table 1 Antibacterial activity of P-PRP against oral microorganisms

\begin{tabular}{|c|c|c|c|c|c|}
\hline \multirow[t]{2}{*}{$\mathrm{N}^{\circ}$ of patient } & \multicolumn{5}{|c|}{ MIC ( $n^{\circ}$ platelets $\left./ \mu \mathrm{l}\right)$} \\
\hline & E. faecalis VRE & E. faecalis VSE & C. albicans & S. agalactiae & S. oralis \\
\hline 1 & $34.475 \pm 13.488$ & $29.550 \pm 11.013$ & $88.650 \pm 22.025$ & $34.457 \pm 13.504$ & $8.618 \pm 3.372$ \\
\hline 2 & $32.500 \pm 19.902$ & $35.750 \pm 17.801$ & $117.000 \pm 29.069$ & $39.000 \pm 14.534$ & $3.250 \pm 1.112$ \\
\hline 3 & $5.738 \pm 2.138$ & $4.303 \pm 1.069$ & $61.200 \pm 20.950$ & $26.775 \pm 10.475$ & $3.346 \pm 1.310$ \\
\hline 4 & $12.488 \pm 3.103$ & $16.650 \pm 6.205$ & $49.950 \pm 12.410$ & $8.305 \pm 3.114$ & $7.650 \pm 2.619$ \\
\hline 5 & $7.613 \pm 5.004$ & $6.831 \pm 5.263$ & $112.500 \pm 27.951$ & $10.937 \pm 4.279$ & $2.734 \pm 1.070$ \\
\hline 6 & $13.956 \pm 6.949$ & $13.956 \pm 6.949$ & $81.200 \pm 27.797$ & $8.881 \pm 3.475$ & $7.612 \pm 2.837$ \\
\hline 7 & $6.581 \pm 1.635$ & $5.850 \pm 2.006$ & $210.600 \pm 52.324$ & $17.550 \pm 6.540$ & $26.325 \pm 6.540$ \\
\hline 8 & $5.375 \pm 3.292$ & $5.913 \pm 2.944$ & $68.800 \pm 23.552$ & $34.400 \pm 11.776$ & $34.400 \pm 11.776$ \\
\hline 9 & $28.425 \pm 10.593$ & $21.319 \pm 5.297$ & $75.800 \pm 25.948$ & $8.290 \pm 3.243$ & $8.290 \pm 3.244$ \\
\hline 10 & $5.611 \pm 2.195$ & $4.809 \pm 1.792$ & $38.475 \pm 14.339$ & $12.825 \pm 4.391$ & $14.428 \pm 3.585$ \\
\hline 11 & $24.200 \pm 8.284$ & $21.175 \pm 8.284$ & $108.900 \pm 27.056$ & $36.300 \pm 13.528$ & $33.275 \pm 16.569$ \\
\hline 12 & $14.000 \pm 4.793$ & $13.125 \pm 6.187$ & $31.500 \pm 7.826$ & $15.750 \pm 3.913$ & $17.500 \pm 10.717$ \\
\hline 13 & $9.075 \pm 4.519$ & $10.725 \pm 5.534$ & $39.600 \pm 14.758$ & $33.000 \pm 20.208$ & $29.700 \pm 7.279$ \\
\hline 14 & $19.906 \pm 11.682$ & $15.641 \pm 11.682$ & $68.250 \pm 25.435$ & $15.640 \pm 7.788$ & $4.976 \pm 1.947$ \\
\hline 15 & $24.850 \pm 9.722$ & $21.300 \pm 7.938$ & $63.900 \pm 15.876$ & $49.700 \pm 19.444$ & $6.212 \pm 2.431$ \\
\hline 16 & $14.850 \pm 10.757$ & $11.550 \pm 4.519$ & $46.200 \pm 18.075$ & $9.900 \pm 3.690$ & $18.150 \pm 9.037$ \\
\hline 17 & $11.375 \pm 5.870$ & $8.750 \pm 5.358$ & $86.800 \pm 53.677$ & $12.250 \pm 4.793$ & $6.125 \pm 2.396$ \\
\hline RANGE & $5.375-34.475$ & $4.303-35.750$ & $31.500-210.600$ & $8.290-49.700$ & $2.734-34.400$ \\
\hline
\end{tabular}


potential. [18] However, Anitua et al. [20] results showed that a further leucocyte dose did not significantly improve the antimicrobial properties of P-PRP. It is also possible that the additional leukocyte content might increase the inflammatory response at the site because of the metalloproteases, pro-inflammatory proteases and acid hydrolases secreted by white blood cells [28].

Bacterial infection is one of the most serious complications impairing wound healing and tissue regeneration. Even when applying strict disinfection, bacteria can infiltrate and colonize the underlying tissues of the wound. The combination of proteolytic enzymes, toxin-rich bacterial exudates and chronic inflammation can alter growth factors and metalloproteinases, thereby affecting the cellular machinery needed for cell proliferation and wound healing $[29,30]$.

Developing approaches and strategies that may help to control or prevent the problem of wound infections would have considerable clinical, social and economic effects.

Our study has shown that P-PRP was active against microorganisms colonizing the oral cavity such as E. faecalis, C. albicans, S. agalactiae and S. oralis, but not against $P$. aeruginosa. Except for E. faecalis and $P$. aeruginosa, PCs have never been tested against such microorganisms.

E. faecalis is associated with different forms of periradicular disease, including primary extraradicular and post-treatment persistent infections. [31] Such microorganism possesses the ability to survive the effects of root canal treatment and persists as a pathogen in the root canals and dentinal tubules of teeth. Implementing methods to effectively eliminate E. faecalis from the dental apparatus is a challenge. We found that P-PRP was active at low platelet concentration ranges (1-2 orders of magnitude lower than the baseline blood values) against this microorganism, while Bielecki et al. [10] observed no activity of platelet concentrate. The reasons for this discrepancy may lie in the different protocol used for platelet concentrate production, which can lead to products with different biological characteristics, or in the different sensibility of the method (Kirby-Bauer discdiffusion method) used to evaluate the susceptibility to platelet concentrate.

Oral candidosis is the most common fungal infection encountered in general dental practice. It manifests in a variety of clinical presentations and can occasionally be refractory to treatment. It is caused by commensal Candida species. While a large majority of healthy individuals harbor strains of Candida intraorally, only selected groups of individuals develop oral candidosis. The most commonly implicated strain is C. albicans, which is isolated in over $80 \%$ of oral candidal lesions. [32] In the present study, we observed that P-PRP was active against $C$. albicans at higher plateletconcentration ranges (same order of magnitude of the baseline blood values) than those effective against the other bacteria tested. This result is consistent with the findings of Tang et al. who tested in vitro antimicrobial activity of seven antimicrobial peptides isolated from human platelets, and noticed that they were more potent against bacteria than fungi [17].

S. agalactiae, S. oralis and P. aeruginosa are some of the many oral biofilm bacteria. We observed that P-PRP was active against $S$. agalactiae and $S$. oralis at platelet concentration ranges similar to the range which inhibited E. faecalis. On the contrary, we found no activity of P-PRP against $P$. aeruginosa at the concentrations used in this experiment. This result is in line with the findings of Bielecki et al. and Burnouf et al., who even observed that platelet concentrate induced growth of this microorganism, suggesting that platelet concentrate may induce a flare-up of infection from $P$. aeruginosa. $[10,11]$ The value of PCs in the presence of a co-existing infection with this bacterium is therefore uncertain.

In our study we also used standard ATCC bacterial strains, which may behave in a way different from isolates, in order to assure reliability of results and reproducibility of experimentation. Results were similar to those obtained with clinical isolates of bacteria.

In addition, we performed a MBC test. We found such test difficult to perform, as P-PRP coagulates at high concentrations. We observed that $C$. albicans was never killed, while the other microorganisms were killed at concentrations 3-4 times the MIC. Further studies are necessary to investigate the potential bactericidal effect of P-PRP. In this study we tested P-PRP in the formulation commonly used in dentistry and oral surgery (that is, plasma fraction activated with $\mathrm{CaCl} 2$ to form a solid coagulum) to assess the potentiality of the use of such preparation in routine clinical practice. Future research may be focused on the analysis of the contribution of individual P-PRP components by employing methods such as separation (e.g. by fractionation according to size) or inactivation (e.g. by exposure to modifying agents, such as specific proteases, or to physical factors, such as heat treatment).

\section{Conclusions}

In conclusion, PCs are safe autologous products, which can be easily prepared during surgery and possess an antibacterial activity. They could be potentially useful substances in the fight against postoperative infections and might represent the linking of osteoinductive and antimicrobial activity. Further research should investigate PCs antimicrobial capacity compared to antibiotics, their exact antibacterial spectrum and prove its efficacy in the in vivo situation. The influence of patients' 
characteristics (sex, age, hematocrit, platelet count, drug assumption, etc....) on antimicrobial activity should be also clarified.

\section{Competing interests}

The authors declare that they have no financial or non-financial competing interest.

\section{Authors' contributions}

LD: Conceived the study, participated in its design and coordination and revised the manuscript. BM: Acquired data, participated in their analysis and interpretation and drafted the manuscript. CV: Acquired data, participated in their analysis and interpretation and drafted the manuscript. ST: Revised the manuscript. MdF: Conceived the study, participated in its design and coordination and revised the manuscript. All authors read and approved the final manuscript.

\section{Author details}

'Laboratory of Clinical Chemistry and Microbiology, I.R.C.C.S. Galeazzi Orthopaedic Institute, Via R. Galeazzi 4, Milan 20161, Italy. ${ }^{2}$ Department of Biomedical Sciences for Health, University of Milan, Milan, Italy. ${ }^{3}$ Department of Biomedical, Surgical and Dental Sciences, I.R.C.C.S. Galeazzi Orthopaedic Institute, University of Milan, Milan 20161, Italy.

Received: 9 November 2012 Accepted: 20 February 2013 Published: 25 February 2013

\section{References}

1. Dohan DM, Choukroun J, Diss A, Dohan SL, Dohan AJ, Mouhyi J, Gogly B: Platelet-rich fibrin (PRF): a second-generation platelet concentrate. Part III: leukocyte activation: new feature for platelet concentrates? Oral Surg Oral Med Oral Pathol Oral Radiol Endod 2006, 101:51-55.

2. El-Sharkawy H, Kantarci A, Deady J, Hasturk H, Liu H, Alshahat M, van Dyke TE: Platelet-rich plasma: growth factors and pro- and anti-inflammatory properties. J Periodontol 2007, 78:661-669.

3. Del Fabbro M, Ceresoli V, Lolato A, Taschieri S: Effect of platelet concentrate on quality of life after periradicular surgery: a randomized clinical study. J Endod 2012, 38:733-739.

4. Cieslik-Bielecka A, Bielecki T, Gazdzik TS, Arendt J, Krol W, Szczepanski T: Autologous platelets and leukocytes can improve healing of infected high-energy soft tissue injury. Transfus Apher Sci 2009, 41:9-12.

5. Everts PA, Devilee RJ, Brown Mahoney C, Eeftinck-Schattenkerk M, Box HA, Knape JT, van Zundert A: Platelet gel and fibrin sealant reduce allogeneic blood transfusions in total knee arthroplasty. Acta Anaesthesiol Scand 2006, 50:593-599.

6. Trowbridge CC, Stammers AH, Woods E, Yen BR, Klayman M, Gilbert C: Use of platelet gel and its effects on infection in cardiac surgery. J Extra Corpor Technol 2005, 37:381-386.

7. Yuan $T$, Zhang $C Q$, Zeng BF: Treatment of chronic femoral osteomyelitis with platelet-rich plasma (PRP): A case report. Transfus Apher Sci 2008, 38:167-170.

8. Anitua E, Alonso R, Girbau C, Aguirre JJ, Murozabal F, Orive G: Antibacterial effect of plasma rich in growth factors (PRGF-Endoret) against Staphylococcus aureus and Staphylococcus epidermidis strains. Clin Exp Dermatol. in press.

9. Álvarez ME, López C, Giraldo CE, Samudio I, Carmona JU: In vitro bactericidal activity of equine platelet concentrates, platelet poor plasma, and plasma against methicillin-resistant Staphylococcus aureus. Arch Med Vet 2011, 43:155-161.

10. Bielecki TM, Gazdzik TS, Arendt J, Szczepanski T, Krol W, Wielkoszynski T: Antibacterial effect of autologous platelet gel enriched with growth factors and other active substances: an in vitro study. J Bone Joint Surg Br 2007, 89:417-420.

11. Burnouf T, Chou ML, Wu YW, Su CY, Lee LW: Antimicrobial activity of platelet (PLT)-poor plasma, PLT-rich plasma, PLT gel, and solvent/ detergent-treated PLT lysate biomaterials against wound bacteria. Transfusion. in press.

12. Moojen DJ, Everts PA, Schure RM, Overdevest EP, van Zundert A, Knape JT, Castelein RM, Cremers LB, Dhert WJ: Antimicrobial activity of plateletleukocyte gel against Staphylococcus aureus. J Orthop Res 2008, 26:404-410
13. Krijgsveld J, Zaat SA, Meeldijk J, van Veelen PA, Fang G, Poolman B, Brandt E, Ehlert JE, Kuijpers AJ, Engbers GH, Feijen J, Dankert J: Thrombocidines, microbicidal proteins from human blood platelets, are C-terminal deletion products of CXC chemokines. J Biol Chem 2000, 275:20374-20381.

14. Wecksler BB, Nachman RL: Rabbit platelet bactericidal protein. J Exp Med 1971, 134:1114-1130.

15. Yeaman MR: The role of platelets in antimicrobial host defense. Clin Infect Dis 1997, 25:951-968.

16. Klinger MH, Jelkmann W: Role of blood platelets in infection and inflammation. J Interferon Cytokine Res 2002, 22:913-922.

17. Tang YQ, Yeaman MR, Selsted ME: Antimicrobial peptides from human platelets. Infect Immun 2002, 70:6524-6533.

18. Dohan Ehrenfest DM, Rasmusson L, Albrektsson T: Classification of platelet concentrates: from pure platelet-rich plasma (P-PRP) to leukocyte- and platelet-rich fibrin (L-PRF). Trends Biotechnol 2009, 27:158-167.

19. Bielecki T, Dohan Ehrenfest DM, Everts PA, Wiczkowski A: The role of leukocytes from L-PRP/L-PRF in wound healing and immune defense: new perspectives. Curr Pharm Biotechnol 2012, 13:1153-1162.

20. Anitua E: Plasma rich in growth factors: preliminary results of use in the preparation of future sites for implants. Int J Oral Maxillofac Implants 1999, 14:529-535.

21. Kraemer BF, Campbell RA, Schwertz H, Cody MJ, Franks Z, Tolley ND, Kahr WH, Lindemann S, Seizer P, Yost CC, Zimmerman GA, Weyrich AS: Novel antibacterial activities of $\beta$-defensin 1 in human platelets: suppression of pathogen growth and signaling of neutrophil extracellular trap formation. PLoS Pathog 2011, 7:e1002355.

22. Levy O: Antimicrobial proteins and peptides of blood: templates for novel antimicrobial agents. Blood 2000, 96:2664-2672.

23. Radek K, Gallo R: Antimicrobial peptides: natural effectors of the innate immune system. Semin Immunopathol 2007, 29:27-43.

24. Blair P, Flaumenhaft R: Platelet alpha-granules: basic biology and clinical correlates. Blood Rev 2009, 23:177-189.

25. Tohidnezhad M, Varoga D, Podschun R, Wruck CJ, Seekamp A, Brandenburg LO, Pufe T, Lippross S: Thrombocytes are effectors of the innate immune system releasing human beta defensin-3. Injury 2011, 42:682-686.

26. Tohidnezhad M, Varoga D, Wruck CJ, Podschun R, Sachweh BH, Bornemann J, Bovi M, Sönmez TT, Slowik A, Houben A, Seekamp A, Brandenburg LO, Pufe T, Lippross S: Platelets display potent antimicrobial activity and release human beta-defensin 2. Platelet 2012, 23:217-223.

27. Tohidnezhad M, Varoga D, Wruck CJ, Brandenburg LO, Seekamp A, Shakibaei M, Sönmez TT, Pufe T, Lippross S: Platelet-released growth factors can accelerate tenocyte proliferation and activate the antioxidant response element. Histochem Cell Biol 2011, 135:453-460.

28. Schnabel LV, Mohammed HO, Miller BJ, et al: Platelet rich plasma (PRP) enhances anabolic gene expression patterns in flexor digitorum superficialis tendons. J Orthop Res 2007, 25:230-240.

29. Falanga V, Grinnell F, Gilchrest B, Maddox YT, Moshell A: Workshop on the pathogenesis of chronic wounds. J Invest Dermatol 1994, 102:125-127.

30. Steed DL, Donohoe D, Webster MW, Lindsley L: Effect of extensive debridement and treatment on the healing of diabetic foot ulcers. Diabetic Ulcer Study Group. J Am Coll Surg 1996, 183:61-64.

31. Stuart CH, Schwartz SA, Beeson TJ, Owatz CB: Enterococcus faecalis: its role in root canal treatment failure and current concepts in retreatment. $J$ Endod 2006, 32:93-98.

32. Farah CS, Lynch N, McCullough MJ: Oral fungal infections: an update for the general practitioner. Aust Dent J 2010, 55:48-54.

doi:10.1186/1471-2180-13-47

Cite this article as: Drago et al: Antimicrobial activity of pure plateletrich plasma against microorganisms isolated from oral cavity. BMC Microbiology 2013 13:47. 